Research article

\title{
Accumulation of tocopherols and tocotrienols during seed development of grape (Vitis vinifera L. cv. Albert Lavallée)
}

\author{
G. Horvath ${ }^{\mathrm{a}, *}$, L. Wessjohann ${ }^{\mathrm{b}}$, J. Bigirimana ${ }^{\mathrm{c}}$, H. Monica ${ }^{\mathrm{c}}$, M. Jansen ${ }^{\mathrm{d}}$, \\ Y. Guisez ${ }^{\mathrm{a}}$, R. Caubergs ${ }^{\mathrm{a}}$, N. Horemans ${ }^{\mathrm{a}}$ \\ ${ }^{a}$ Research Group of Plant Physiology, Department of Biology, University of Antwerp, Groenenborgerlaan 171, 2020 Antwerp, Belgium \\ ${ }^{\mathrm{b}}$ Leibniz Institute of Plant Biochemistry, Weinberg 3, D-06120 Halle (Saale), Germany \\ ${ }^{\mathrm{c}}$ Research Group of Plant Pathology, University of Ghent, Coupure Links, 9000 Ghent, Belgium \\ ${ }^{\mathrm{d}}$ Department of Zoology, Ecology and Plant Science, University College Cork, Butler Building, Distillery Field, North Mall, Cork, Ireland
}

Received 6 October 2006; accepted 9 October 2006

Available online 27 October 2006

\begin{abstract}
Tocopherols and tocotrienols are present in mature seeds. Yet, little is known about the physiological role and the metabolism of these compounds during seed development. Here we present data on tocopherol and tocotrienol accumulation during seed development in Vitis vinifera L. cv. Albert Lavallée (Royal). This species was chosen for its ability to synthesize both tocopherols and tocotrienols. It is shown here for the first time that during seed development there are significant differences in localization and accumulation kinetics of tocopherols and tocotrienols. Tocopherols are found homogeneously dispersed throughout all tissues of the seed, in concentrations ranging from 20 to $100 \mu \mathrm{g}$ tocopherol per $\mathrm{g}$ dry weight. Tocopherol levels decrease gradually during seed development. In contrast, tocotrienols are only found in the endosperm of the seeds, accumulating in a sigmoid fashion during the maturation period of seed development. Tocotrienol levels were found to be (54 \pm 7.4 ) $\mu \mathrm{g} / \mathrm{g}$ dry seed in 90-day-old seeds of $V$. vinifera L. Furthermore, tocotrienol biosynthesis is demonstrated in these seeds during tocotrienol accumulation and in an endosperm fraction isolated at 75 days after flowering.
\end{abstract}

(C) 2006 Elsevier Masson SAS. All rights reserved.

Keywords: Tocopherol; Tocotrienol; Vitamin E; Seed development; Tocotrienol biosynthesis; Vitis vinifera L

\section{Introduction}

Tocopherols and tocotrienols are known under the generic name vitamin E, and the chemical name tocochromanols [15]. Tocopherols appear to be universal constituents of all higher plants [31] whereas tocotrienols are thought to be present in a limited number of species and tissues, including in cereal seeds [20], fruits of Elaeis guineensis Jacq. [17] and in the latex of Hevea brasiliensis (Willd. ex A. Juss.) Müll. Arg. [6]. In addi-

Abbreviations: DAF, days after flowering; GGPP, geranylgeranyl pyrophosphate; HGGT, homogentisate geranylgeranyl transferase; HPT, homogentisate phytyl transferase; IPP, isopentenyl pyrophosphate; PhyPP, phytyl pyrophosphate; SAM, S-adenosyl methionine.

${ }^{*}$ Corresponding author. Tel.: +32 3265 3421; fax: +32 32653417.

E-mail address: gyorgy.horvath@ua.ac.be (G. Horvath). tion to its antioxidant function, vitamin $\mathrm{E}$, has been suggested to play a role in a range of different physiological phenomena including plant growth and development, senescence and to interact with the signal cascades that convey abiotic and biotic stress signals [18]. However, some of these functions are currently under discussion as the vegetative growth of mature Arabidopsis thaliana L. mutants (vte1 and vte 2), deficient in tocopherol metabolism, is not significantly altered compared to wild type plants [13]. Studying the same mutants, it was recently demonstrated that tocopherols are vital in preventing lipid peroxidation in seeds, ensuring seed longevity and healthy germination [25].

Biochemical aspects of the biosynthesis of tocopherols were recently reviewed by Hofius and Sonnewald [13] and currently the genes encoding for the enzymes of this biosynthetic pathway are being identified [4,24,26,27] and heterologously 
expressed [8,35]. In contrast to the tocopherols, tocotrienol biosynthesis is not fully understood. The enzyme homogentisate geranylgeranyl transferase (HGGT), catalyzing the formation of tocotrienols, was characterized based on its homology with the homogentisate phytyl transferase (HPT) gene [2]. Overexpression of HGGT in A. thaliana (L.) Heynh. and Nicotiana tabacum L. resulted in elevated tocopherol and tocotrienol levels [2]. Intriguingly, co-expression of the Saccharomyces cerevisiae (yeast) prephenate dehydrogenase gene and the $A$. thaliana $p$-hydroxyphenylpyruvate dioxygenase coding sequence also induced massive accumulation of tocotrienols in leaves of N. tabacum L. (tobacco) plants [22]. In general, it seems that increasing the homogentisate pool can lead to elevated tocotrienol levels [16]. The progress in tocochromanol engineering is recently discussed [34].

The kinetics of tocopherol accumulation during leaf growth and development have been described. During the early stages of germination the concentration of tocopherol, mostly the $\alpha$ form, increases in developing primary leaves of monocotyledonous grasses like Triticum vulgare L. [11], Triticum aestivum L., Hordeum vulgare L., Secale cereale L. and Oryza sativa L. (Horvath, unpublished results). In dicots like soybean and pinto beans, an initial increase in $\alpha$-tocopherol levels seen at the onset of germination was found to be followed by a decline $[23,32]$. Immature chloroplasts isolated from 2-week-old Spinacia oleracea $\mathrm{L}$. seedlings demonstrate a high tocopherol biosynthetic potential [29]. Tocopherol concentrations in older seedlings do not rise any further, presumably due to a combination of lower de novo synthesis and regeneration by, for instance, ascorbate $[12,18]$. Chloroplasts isolated from mature leaves showed a very low biosynthesis of tocopherols [1]. Indeed, leaf aging is accompanied by loss of $\alpha$-tocopherol in Fagus sylvatica [10]. A transient peak of $\alpha$ - and $\gamma$-tocopherol has been observed in early leaf senescence in $H$. vulgare L. and Festuca pratensis Huds. [19]. The differences in tocopherol biosynthesis activity possibly originate from a difference in precursor availability. In developing chloroplasts isoprenoids (IPP), the precursors of the tocopherol side chain, are actively synthesized, whereas in mature chloroplasts IPP are imported from the ER-cytosolic mevalonate pathway [28]. During the reproductive stage tocopherols have been shown to be associated with the chloroplast to chromoplast transition in fruits [3].

In contrast to the ubiquitous tocopherols, tocotrienols are mainly found in seeds. Their probable function is the prevention of oxidation of fats and stored oils [2,18,28]. A transient peak of tocotrienols is also present in germinating seedlings of T. aestivum, $H$. vulgare L., O. sativa L., S. cereale L., Vitis vinifera $\mathrm{L}$. (Horvath, unpublished results). While numerous studies have highlighted the accumulation of tocopherols in leaf tissues, very little is known about the accumulation and role of tocopherols and tocotrienols in seeds. Yet, this appears to be of major significance as tocopherol mutants were specifically altered in their seed physiology [25]. Tocotrienols were found in endosperm of imbibed, full-grown seeds of T. aestivum L. by Hall and Laidman [11] who performed tracer experiments on germinating seedlings in order to study tocopherol metabolism. Con- centrations of $15 \mu \mathrm{g} \alpha$-tocotrienol/150 grains and $49 \mu \mathrm{g} \beta$ tocotrienol/150 grains at the onset of germination were reported. Tocotrienol levels declined during germination. The endosperm fraction of the full-grown seeds was not capable to introduce labeled precursors to form either tocopherol or tocotrienol during this experiment, indicating a lack of biosynthetic capacity. The embryo contained no tocotrienols.

In this study we address the enigma of biosynthesis, accumulation and physiological role of tocotrienols and tocopherols in the oil of full-grown seeds. It analyses in detail the accumulation process of tocotrienols and tocopherols during seed development of $V$. vinifera L. Alphonse Lavallée (Royal). The relationship between the synthesis of tocotrienols and the synthesis of tocopherols, during different phases of seed development is discussed.

\section{Results}

\subsection{Seed development}

$V$. vinifera L. cv. Alphonse Lavallee (Royal) grape and seed development was monitored during 2 subsequent years (2002, 2003). Grape development is well described [5], and morphological parameters can be used to identify the different growth stages. Yet, these morphological markers have been developed to describe grape development of cultivars used in wine production. Since, we have used a Table grape in this study, grape development was meticulously monitored and described, by us. Our criteria for differentiating the different developmental stages of berry development were: days after flowering (DAF), berry size [33], morphology of the berry and the seed $[7,21]$, changes in seed fresh weight and dry weight [36]. The cited references were used for confirmation and terminology.

During the first sigmoidal growth period (from bloom to $50 \mathrm{DAF}$ ) (Fig. 1) grapes evolved from perfect, small green

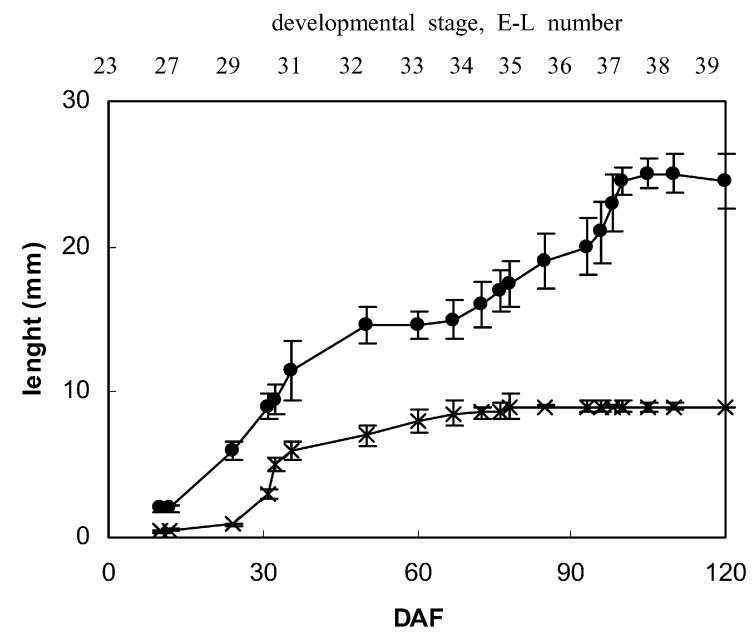

Fig. 1. Grape size (circles) as recorded during each developmental stage ( $N=20$, standard error (S.E.) is shown) is given as marker for grape development. Furthermore seed size is shown (crosses). Both DAF and development stage as describe by Coombe [5] are indicated. 
spheres to $15 \mathrm{~mm}$ green spheres. Seeds changed visually during this first phase. At first the seeds remained small in contrast to the growing grapes. At this point the nucellus, an enigmic form of endosperm, formed in the cavities created by the growing integumentum. At the end of the first sigmoidal growing period, the berry growth rate slows down while the seeds overtake the pace of grape growth. The berry showed an apparent 16 days rest in development (from 50 to $66 \mathrm{DAF}$ ), called lag phase. Despite the apparent rest in the grape development, seeds were very actively growing to full size $(9 \mathrm{~mm})$ and maturing, seeds became hard but remained green. Cellular endosperm was replacing the nucellus. After the lag phase, berry growth restarted and ripening set in, marked by the onset of blue pigmentation (75 DAF). The seed continued maturing, and became red-brown. Endosperm completely overtook the nucellus tissue. Grapes then reached harvest maturity at 110-120 DAF.

\subsection{Tocochromanol content during development}

\subsubsection{Tocopherols}

In order to analyze the chromanols, samples were taken from each developmental stage of both grapes and seeds. Fig. 2 (panels V1, V2 and V3), shows the tocopherol content in pericarp, mesocarp and seeds during development. Tocopherols are rather homogeneously distributed in all tissues of grape. On average, concentrations range from 5 to $100 \mu \mathrm{g}$ tocopherol per g dry weight. As seen in Fig. 2, panel V1, total tocopherol concentrations found in the pericarp at the start of grape development (10 DAF) are $(48.4 \pm 5.8) \mu \mathrm{g}$ tocopherol per g dry weight and drop sharply to $(10.3 \pm 1.1) \mu \mathrm{g}$ tocopherol per $g$ dry weight by the end of grape development (120 DAF). The $\alpha$-tocopherol form is prevalent in the pericarp. Starting at veraison (75 DAF), low amounts of $\gamma$ tocopherol could be detected in addition to $\alpha$-tocopherol. The mesocarp, as shown in Fig. 2, panel V2, contained threefold lower levels of tocopherol. The total tocopherol concentrations in the mesocarp at the start of development (10 DAF) are $(18.6 \pm 1.9) \mu \mathrm{g}$ tocopherol per $\mathrm{g}$ dry weight and they drop to $(3.8 \pm 0.3) \mu \mathrm{g}$ tocopherol per $\mathrm{g}$ dry weight by the end of the experiment (120 DAF). In the mesocarp, the $\alpha$-tocopherol form is prevalent, just like in the pericarp. In contrast to the absence of $\gamma$-tocopherol in pericarp tissue (Fig. 2, panel V1) at the early stages of seed development (10 DAF), small amounts of $\gamma$-tocopherol are already found in the mesocarp at this stage (Fig. 2, panel V2). $\gamma$-Tocopherol content further increases with development. The levels of the $\beta$ - and $\delta$ variants of tocopherol were always below detection limit. For grape seeds, as shown in Fig. 2, panel V3, the highest tocopherol levels are found at early stages of development with $(97.6 \pm 3.8) \mu \mathrm{g}$ tocopherol per $\mathrm{g}$ dry weight. Following is a short stationary phase with $(43.4 \pm 5.6) \mu \mathrm{g}$ tocopherol per g dry weight (30-60 DAF), and at the onset of desiccation (80-120 DAF) tocopherol levels gradually declined to $(20.4 \pm 0.2) \mu \mathrm{g}$ tocopherol per $\mathrm{g}$ dry weight, which is about $25 \%$ of the initial concentration. As shown in Fig. 2, panel $\mathrm{V} 3$, the prevalent form in all but the youngest of seeds, is the
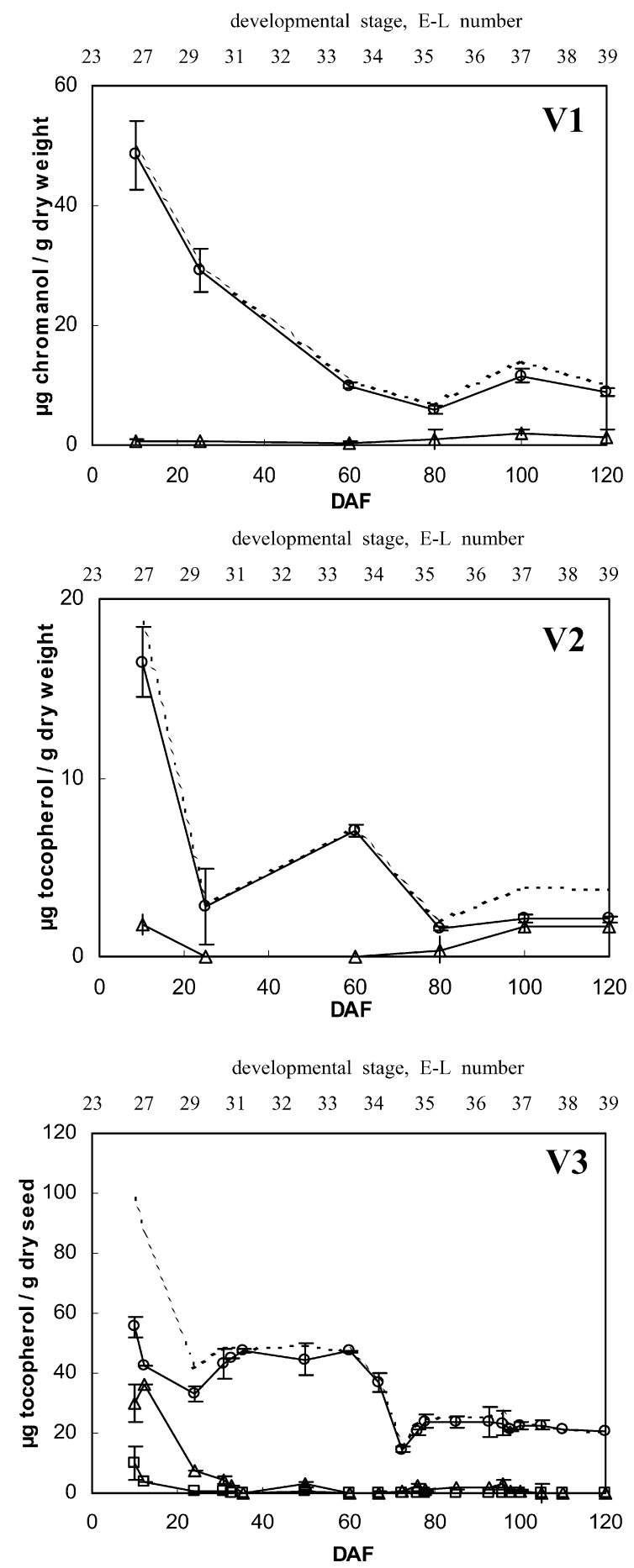

Fig. 2. Tocopherol concentrations during the reproductive phase of grapes of $V$. vinifera L. The developmental process is expressed as DAF and growth stages are indicated by the E-L scale. Grapes were divided in pericarp (panel V1), mesocarp (panel V2) and seeds (Panel V3). $\alpha$-Tocopherol (circles), $\beta$ tocopherol (crosses), $\gamma$-tocopherol (triangles) and $\delta$-tocopherols (squares) are shown. Total tocopherol concentration is indicated as a dashed line $(N \geq 5$ and S.E. is given).

$\alpha$-form. During the first developmental stages, shortly after bloom, the $\gamma$-form $(50 \%)$ and $\delta$-form $(10 \%)$ are also found in large amounts. These forms decrease rapidly during further development. 


\subsubsection{Tocotrienols}

In $V$. vinifera L. tocotrienols are only present in the seeds. In sharp contrast to the tocopherols, tocotrienol levels were below the detection limit at the onset of seed growth as shown in Fig. 3. Significant levels of tocotrienols were first present at $50 \mathrm{DAF}$. Tocotrienol levels gradually increased during development to reach a maximum value of $(54 \pm 7.4) \mu \mathrm{g} / \mathrm{g}$ dry weight. Accumulation continued until shortly after veraison (85 DAF). During this period both seed and endosperm reached maturity. We found that $\gamma$-tocotrienol is most abundant $(65 \%)$ while $\alpha$-tocotrienol values are ca. twofold lower. The concentrations of the $\beta$ - and $\delta$-forms were very low. Thus, all tocotrienols accumulated within a time span of 35 days.

\subsubsection{Total oil content}

Seeds of $V$. vinifera $\mathrm{L}$. were monitored during the complete developmental process for total oil content. It is shown in Fig. 4 that a correlation $\left(R^{2}=0.87\right)$ between total tocotrienol content and total oil content is evident. This correlation is completely absent when total tocopherol content and total oil content are compared.

\subsubsection{Localization of tocotrienols in endosperm}

To further determine the localization of tocotrienols in seeds of $V$. vinifera, these were dissected in four different fractions. Grape seeds were first "perpendicularly" cut in two. The "side" with the embryo contained less tocotrienols than the non-embryo side. Further seed dissection showed that only very low concentrations of tocotrienols are present in the hard palisade layer fraction, described as outer integument. However, the endosperm tissue was enriched in tocotrienols (Fig. 5). As the outer integumentum is an invaginated structure that is in close contact with the endosperm tissue, it is not possible to completely separate both tissues. Therefore

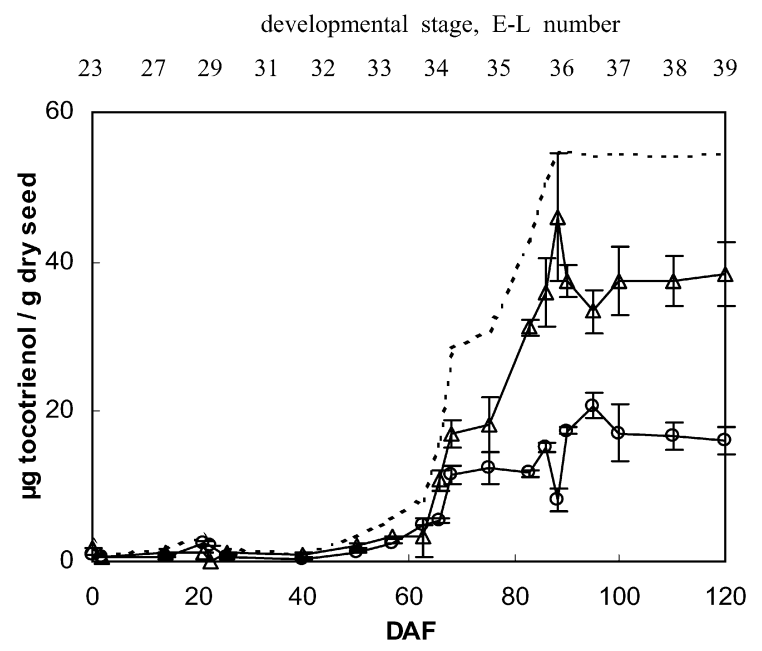

Fig. 3. Tocotrienol concentrations in seeds during the reproductive phase of $V$. vinifera $\mathrm{L}$. The developmental process is expressed as DAF and growth stages are indicated by the E-L scale for grapes. $\alpha$-Tocotrienol (circles), $\gamma$ tocotrienol (triangles) and $\delta$-tocotrienols (squares) are shown. Total tocotrienol concentration is indicated as a dashed line ( $n \geq 5$ and S.E. is given). the low levels of tocotrienols found in the integumentum fraction could well be contamination from endosperm tissue. In grape seeds, roughly two seeds out of 10 are abnormal, they show "empty-seededness". These seeds are referred to as floaters [7]. The endosperm of floaters degenerates after veraison or is not even formed during seed morphogenesis, leaving the seed endospermless. As shown in Fig. 5, no tocotrienols can be detected in the floater seeds.

\subsection{Biosynthesis of tocotrienols in $V$. vinifera $L$}

To measure tocotrienol biosynthesis, feeding experiments were performed using $2 \mu \mathrm{Ci}{ }^{3} \mathrm{H}$-GGPP and dissected seeds from grapes at six different developmental stages (full bloom (50\% cap fall; 0 DAF), setting (18 DAF), bunch closure (42 DAF), during lag phase (62 DAF), at veraison (78 DAF), and berries harvest ripe (105 DAF)). The tocochromanol biosynthesis assay has been optimized for this specific $V$. vinifera L. cultivar as we reported earlier [14]. Discrimination between tocotrienol and tocopherol biosynthesis is possi-

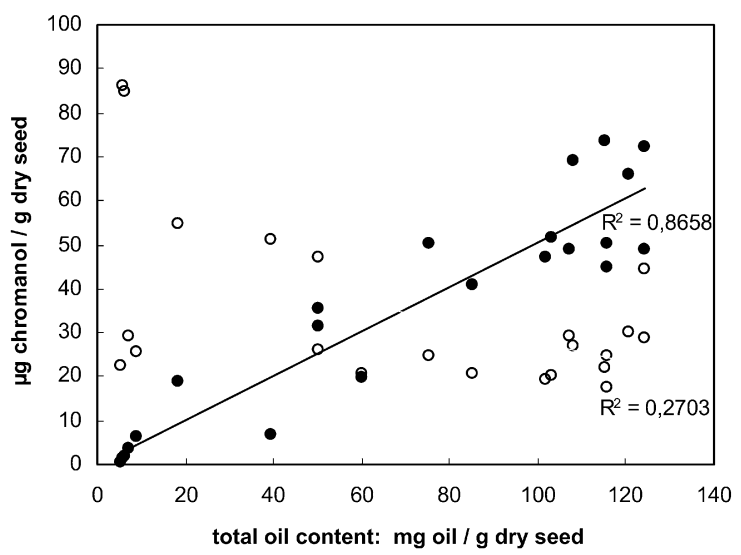

Fig. 4. Seeds of $V$. vinifera L. Albert Lavallée (Royal) monitored during the complete developmental process (from anthesis to desiccation) showed a correlation between total tocotrienol content and total oil content $\left(R^{2}=0.87\right)$, shown as full circles. This correlation is completely absent when comparing total tocopherol content and total oil content $(n \geq 5)$.

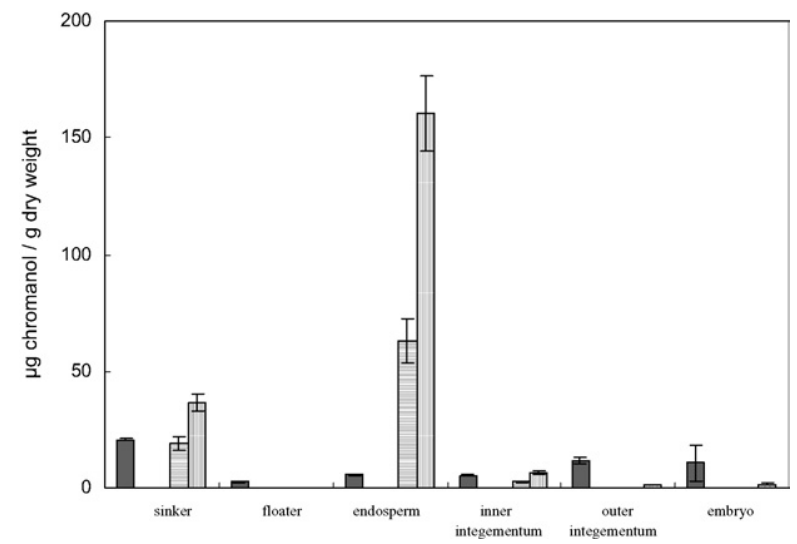

Fig. 5. Tocopherol and tocotrienol concentrations in distinct seed types or dissected tissue of seeds of $V$. vinifera L. $\alpha$-Tocopherol (dark gray bars), $\alpha$ tocotrienol (open bars with horizontal stripes), $\gamma$-tocotrienol (Open bars with vertical stripes) are shown ( $n \geq 5$, S.E. is shown). 
$\begin{array}{llllllllllll}23 & 27 & 29 & 31 & 32 & 33 & 34 & 35 & 36 & 37 & 38 & 39\end{array}$
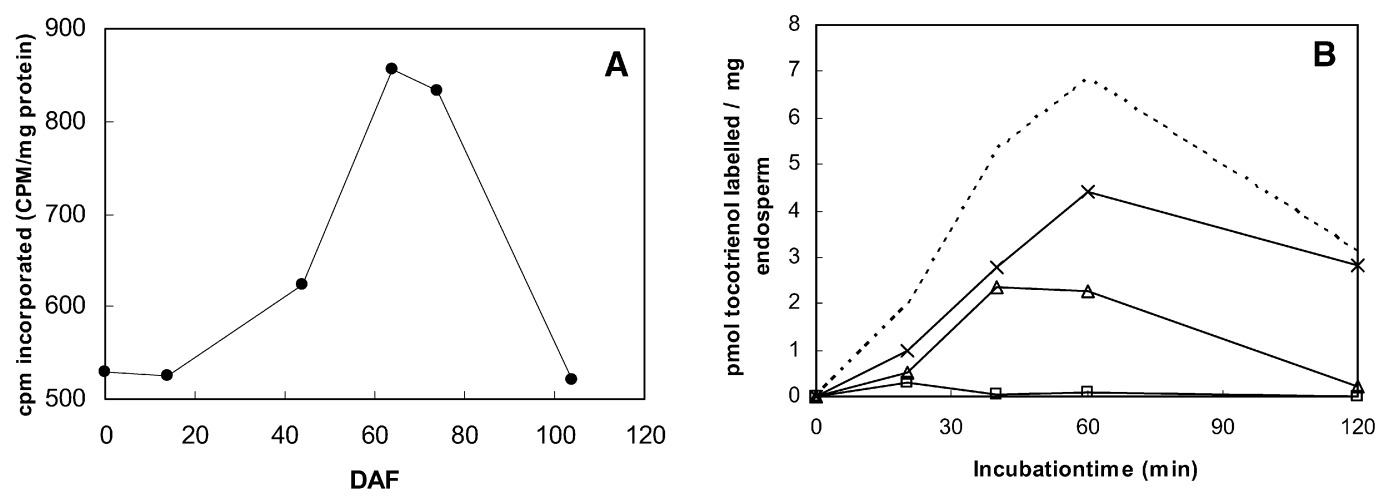

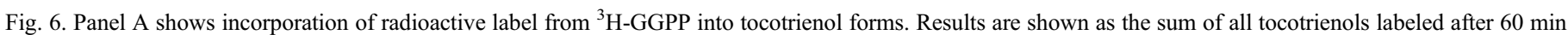

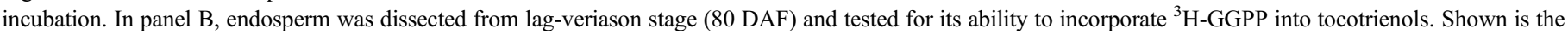

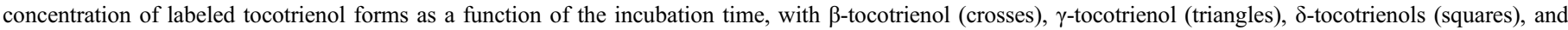
total tocotrienol radiolabel concentration indicated as a dashed line.

ble by, respectively, omitting or adding NADPH, a necessary cofactor for the conversion of GGPP to PhyPP. With this assay, we found no labeling of tocopherol molecules during any stage of seed development. These data indicate that tocopherol synthesis was below our detection limit during $V$. vinifera seed development. In contrast, as shown in Fig. 6, tocotrienol molecules were labeled at 20 DAF and gradually increased until $62 \mathrm{DAF}$. As ripening commenced (105 DAF), grapes started to lose the potential to incorporate label in tocotrienols. The strongest labeling is found in seeds in the lag phase (62 DAF), corresponding to $875 \mathrm{cpm}$ incorporated label. It should, however, be noted that some background labeling of tocotrienols was found during all developmental stages, but not in control experiments with inactivated (boiled) extracts. Tocotrienol biosynthesis was further studied using endosperm tissue dissected from seeds of $V$. vinifera at the end of the lag phase (75 DAF). This endosperm could incorporate ${ }^{3} \mathrm{H}-\mathrm{GGPP}$ into the tocotrienol fractions. Fig. 6, panel B shows the linear formation of labeled tocotrienols, indicating a specific activity of $7 \mathrm{pmol}$ of labeled total tocotrienol/hour $\times \mathrm{mg}$ endosperm, up to ca. 1 hour. Longer incubation times were hampered by the breakdown of the tocotrienols in the assay. Radioactive label was mainly found in $\beta$-tocotrienols, and to a lesser extent in $\gamma$-, and $\delta$-tocotrienols. In an identical experiment with a similar endosperm fraction, however, with NADPH added, again no tocopherol molecules were labeled.

\section{Discussion}

Only a limited number of plants have the ability to simultaneously produce both tocopherols and tocotrienols[31]. Tocopherols are uniformly distributed over all the tissues tested, during seedling, vegetative (leaf, stem) and reproductive stages (bud, flowers, grape, seed, grain). Our data confirm that tocopherol is a ubiquitous plant component. Literature sources indicate that the major tocopherol form in leaf tissues is the $\alpha$-form ( $>90 \%)$. Seeds and oils extracted from seeds were reported to contain predominantly the $\gamma$-tocopherol (> 70\%) form [28]. In contrast to this, we found that seeds of $V$. vinifera $\mathrm{cv}$. Albert Lavallée (Royal) contain primarily the $\alpha$-form of tocopherol. Some $\gamma$-tocopherol was found in the epidermis and the pericarp during the very early stages of seed development as well as the late stages of grape development. As $\alpha$-tocopherol is considered the ultimate step in tocopherol biosynthesis [28], our data indicate that the complete tocopherol pathway is present and active in our plants.

In strong contrast to tocopherols, tocotrienols were only found in seeds of $V$. vinifera, except for a small transient peak in young seedlings (data not shown). The major tocotrienol form found in the seeds is the $\gamma$-form $(\geq 60 \%)$, while the remainder is in the $\alpha$-form. This indicates that conversion was not complete, as the presumable end product, i.e. $\alpha$ tocotrienol is not the predominant product.

Tocopherols and tocotrienol levels were followed throughout development of grapes seeds. In all tested tissues a stepwise decline in tocopherol concentrations was measured as seeds developed. At the end of grape development, tocopherols had reached a constant concentration which was roughly five times lower compared to that one at the onset of seed development. Previously, it had been shown that after longer desiccation and even oil extraction, tocopherol concentrations remain constant in $V$. vinifera $L$. seeds [14]. We noted an analogy with vegetative tissue development, as highest tocopherol concentrations are often found in the early developmental stages, while declining during maturation [28].

Tocotrienols were, in contrast to the tocopherols, not present at the start of seed development. Here we show for the first time that the tocotrienols found in the grape accumulate in a sigmoid manner during a short spell of the seed development programme. Accumulation of tocotrienol molecules starts at the beginning of the lag phase (50 DAF) and continues during veraison, a period of just 35 days with a highest 
accumulation rate at 70 DAF. Apparently, all tocotrienols found in $V$. vinifera $\mathrm{L}$. are formed in this time frame, indicating a closely regulated temporal expression pattern correlating with the lag phase of grape development. The lag phase of grape development is the final expansion phase of the seed. At this point, we do not know how this window of tocotrienol synthesis is regulated.

As discussed earlier, in the maturation stage the accumulation of storage compounds like starch, oils, and proteins takes place. The possible relation between oil accumulation and tocotrienol accumulation could be explained by the proposed function of tocotrienols in protecting storage oil from oxidative damage [2]. Indeed, we have found that tocotrienols are uniquely restricted to endosperm tissue of grape as indicated by the clear enrichment of dissected endosperm. The presence of endosperm is also clearly a necessary precondition for tocotrienol formation because endospermless seeds, that are otherwise physiologically identical to normal seeds [7], contain no detectable amounts of tocotrienols.

An analogy is observed between the accumulation of tocotrienols in grains of the monocotyledonous $H$. vulgare $\mathrm{L}$. (Poaceae) [9] and the dicotyledonous $V$. vinifera $\mathrm{L}$ results presented here. Seemingly, H. vulgare L. shows tocotrienol formation starting with seed formation. For $V$. vinifera $\mathrm{L}$. this process is postponed to a later developmental stage but coincides with the disappearance of the nucellus and appearance of the endosperm. It seems that in both cases tocotrienol formation is correlated with endosperm formation. The results are differing in that for $H$. vulgare $\mathrm{L}$. tocotrienols are not solely restricted to endosperm but are also found in pericarp.

Tocotriënol biosynthesis in $V$. vinifera L., studied by feeding experiments with labeled precursor, is active during the time span that tocotrienols accumulation takes place. Although biosynthesis measurements were close to the detection limit, a Gaussian curve was found. An explanation for the background value found in all samples except the boiled control could be the potential of these enzymes to work in vitro, whereas in vivo function is inhibited. Alternatively, a nonspecific prenyltransferase could catalyze the conversion of the radioactive precursors to tocotrienols.

For tocopherols, biosynthesis is below the detection limit in seed extracts. It has been reported that tocopherol biosynthesis can only be measured in purified chloroplasts [30], therefore lack of synthesis in seed extracts is consistent. Indeed, using the same biosynthesis assay, we were able to label tocopherols using chloroplasts isolated from leaves of $S$. oleracea, proving the usability of our assay (data not shown).

Tocotrienol biosynthesis was further examined by feeding dissected endosperm (72 DAF) with the ${ }^{3} \mathrm{H}$-GGPP. Endosperm had a total tocotrienol biosynthetic activity of 7 pmol labeled tocotrienol/( $\mathrm{h} \times \mathrm{mg}$ protein) which was linear for about 1 hour. After that time, incorporation decreased. Taken together with the fact that tocotrienols are strongly enriched in endosperm fractions of both grape and T. aestivum, these data support endosperm biosynthesis and localization of tocotrie- nols in plants. Further in silico analysis of the promoter sequence of the rice HGGT gene recently identified by Cahoon et al. [2] with target prediction programs (PLACE) shows a prolamin box in the gene promoter, a necessary indication for endosperm-specific gene expression [37]. Remarkably, the tocotrienol forms formed in the different experiments cannot be reconciled. In vivo experiments clearly show that $\gamma$ tocotrienol is the major form throughout the whole growth phase with $\alpha$-tocotrienol a close second, $\beta$ - and $\delta$-forms were below detection limit. In contrast the labeling experiments showed formation of mainly $\beta$ - and $\gamma$-tocotrienols and a small amount of $\delta$-tocotrienol. $\alpha$-Tocotrienol was not detected. This discrepancy was also reported by Soll et al. [29] studying in vitro different tocopherol forms in young leaves of $S$. oleracea L. Possibly, activity of methyl transferases involved in the transformation of $\beta$ - and $\gamma$-tocotrienols to, respectively, $\delta$ - and $\alpha$-tocotrienols could be lost during extraction and/or incubation during the in vitro experiments. Another explanation could be that SAM is present in limiting amounts.

In conclusion, our data show significant differences between tocotrienol and tocopherol metabolism and accumulation in developing seeds. (1) Tocotrienols accumulate specifically during a short window of the seed development programme, whereas tocopherol levels decrease with seed age, tocotrienol accumulation largely coincides with oil accumulation, therefore our findings suggest a role for them in the protection of storage oil against oxidative stress. (2) Tocotrienols are mostly in $\gamma$-form, while tocopherols are found in the $\alpha$-form. (3) The presence of tocotrienols is restricted to endosperm tissue whereas tocopherols are found ubiquitously distributed throughout all tested tissues. (4) Finally, in vitro biosynthetic activity was found during the accumulation of tocotrienols, particularly in the isolated endosperm fraction.

\section{Methods}

\subsection{Plant material}

Grapes of $V$. vinifera L. cv. Alphonse Lavallé (Royal) were obtained from a commercial grape grower (grape producers Soniën; Brusselsesteenweg 538, Overijse 3090, Belgium) and this involved multiple sampling sessions over 2 subsequent years. The grapes were grown under optimal conditions. Soil moisture was mechanically controlled. Due to canopy growth, bunches were always protected from direct sunlight and leaves were optimally light exposed. On 50\% cap-fall, buds were labeled in order to determine DAF. During sampling whole bunches of different developmental stages were cut and stored either on ice or liquid nitrogen. Seeds stored in liquid nitrogen were first lyophilized $(133 \times$ $10^{-3} \mathrm{mBar},-42^{\circ} \mathrm{C}, 2$ days) and subsequently used for analytical determinations of chromanols. Seeds stored on ice were used immediately, for labeling experiments after manual removal of the flesh. In order to asses grape development, we 
recorded grape size, DAF, volume, dry weight, color and grape and seed morphology.

\subsection{Chromanol analysis}

Chromanols were extracted from lyophilized material, which was ground in liquid nitrogen in the presence of hexane. DMT (5 ppm) was used as an internal standard. Normally four subsequent extractions were performed, pooled and dried under vacuum (Speed-Vac). Separation of the different chromanol forms was achieved by HPLC, using a normal phase system. All components were eluted isocratically $(1 \mathrm{ml} / \mathrm{min}$ ) with $6 \%$ THF in hexane in $25 \mathrm{~min}$ on a Particil Pac column (5 $\mu \mathrm{m}$, length $250 \mathrm{~mm}$, i.d. $4.6 \mathrm{~mm}$ (Alltech)). Two on line detectors were used: fluorescence detection (Shimadzu RF-10A, ex. $=295$, em. $=325$ ) for its high sensitivity, and a DAD (Shimadzu SPD-M10avp, 200-600nm) for peak purity determination. Data were analyzed with the Class-vp 6.0 software package (Shimadzu).

\subsection{Assay conditions}

Labeling experiments were performed on seeds or on dissected endosperm. The seeds were used after removal of all other parts of the fruit and washed shortly in ice cold grinding buffer 1 (15 mM tricine ( $\mathrm{pH} 7.6), 0.6 \mathrm{M}$ sucrose, $5 \mathrm{mM}$ DTT, $1 \mathrm{mM} \mathrm{MgCl} 2,5 \mathrm{mM}$ EDTA, $0.2 \%$ BSA, $0.3 \%$ (w/v) nonsoluble PVPP, $5 \mathrm{mM}$ DTT and $0.5 \mathrm{mM}$ PMSF). Typically, $10 \mathrm{~g}$ of seeds were used for each time point of a kinetic analysis. The seeds were ground for $30 \mathrm{~s}$ with an IKA Ultra Turax homogenizer in grinding buffer 1. Care was taken to keep the homogenate on ice. The homogenate was filtered through miracloth and the filtrate was used as the whole seed-extract in our labeling essays. For experiments on endosperm, seeds were dissected with a forceps under a Zeiss Sterni 2000 binocular microscope, endosperm fragments were immediately placed in grinding buffer $2(50 \mathrm{mM}$ Hepes $\mathrm{pH}$ (6.8), $330 \mathrm{mM}$ sorbitol, $1 \mathrm{mM} \mathrm{MgCl}_{2}, 1 \mathrm{mM} \mathrm{MnCl}_{2}$ ) and homogenized thoroughly in a potter. Extracts of either full seeds or endosperm (three replicates, $5 \mathrm{mg}$ protein each) were incubated at $30{ }^{\circ} \mathrm{C}$ in incubation mixture $(10 \mathrm{mM}$ Hepes $(\mathrm{pH}$ 7.6), $4 \mathrm{mM} \mathrm{MgCl}$, $2 \mathrm{mM} \mathrm{MnCl}, 0.1 \mathrm{mM} \mathrm{NaF}, 0.5 \mathrm{mM}$ ATP, $10 \mathrm{mM} \mathrm{NaHCO}, 70 \mu \mathrm{M}$ SAM, $180 \mu \mathrm{M}$ GGPP, $180 \mu \mathrm{M}$ Hom, $2 \mathrm{mM}$ cyclodextrine) together with the radioactively labeled precursor $\left[1(n)-{ }^{3} \mathrm{H}\right] \mathrm{GGPP}(0.2 \mu \mathrm{Ci})$. Samples were taken at $20 \mathrm{~min}$ intervals. The extract was added last, in order to insure an osmotic shock. Incubation was stopped by adding $5 \mathrm{ml} \mathrm{EtOH}$ and immediate extraction with $15 \mathrm{ml}$ hexane. The mixture was vigorously vortexed for $5 \mathrm{~min}$ and three times re-extracted. The pooled solvent fraction was placed in a rotary evaporator until completely dry, dissolved in hexane and applied to the HPLC system described above. HPLC fractions were collected and measured in a Packard liquid scintillation counter with OCS liquid scintillation cocktail. The three time points were plotted in order to evaluate the linearity and to allow the determination of the enzymatic activity.

\subsection{Total oil content}

Total oil content of seeds was determined by extraction of freeze dried $\left(-40{ }^{\circ} \mathrm{C}, 121 \mathrm{mbar}, 48 \mathrm{~h}\right)$ seeds grinded in hexane and subsequent weighing.

\subsection{Protein determination}

Protein concentration was measured according to the method of Bradford (1976) using BSA as a standard.

\section{Acknowledgements}

Financial support by the IWT (instituut ter bevordering van wetenschappelijk technologisch onderzoek in Vlaanderen) is thankfully acknowledged. Eddy Biebaut is acknowledged for his skilful technical assistance, and the numerous HPLC analyses. N.H. is a post-doctoral researcher at the scientific fund for research (FWO)-Flanders.

\section{References}

[1] H. Bickel, L. Palme, G. Schultz, Incorporation of shikimate and other precursors into aromatic amino acids and prenylquinones of isolated spinach chloroplasts, Phytochemistry 17 (1978) 119-124.

[2] E.B. Cahoon, S.E. Hall, K.G. Ripp, T.S. Ganzke, W.D. Hitz, S.J. Coughlan, Metabolic redesign of vitamin E biosynthesis in plants for tocotrienol production and increased antioxidant content, Nat. Biotechnol. 21 (2003) 1082-1087.

[3] B. Camara, A. D'Harlingue, Demonstration and solubilisation of S-adenosylmethionine:gamma-tocopherol methyltransferase from Capsicum chromoplasts, Plant Cell Rep. 4 (1985) 31-32.

[4] E. Collakova, D. DellaPenna, Isolation and functional analysis of homogentisate phytyltransferase from Synechocystis sp. PCC 6803 and Arabidopsis, Plant physiol. 127 (2001) 1113-1124.

[5] B.G. Coombe, Adoption of a system for identifying grapevine growth stages, Aust. J. Grape Wine Res. 1 (1995) 104-110.

[6] J. D'Auzac, J.L. Jacob, H. Chrestin, Physiology of Rubber Tree Latex, CRC Press, Inc., Boca Raton, Florida, 1989.

[7] A. Ebadi, M. Sedgley, P. May, B.G. Coombe, Seed development and abortion in Vitis vinifera L. cv. Chardonnay, Int. J. Plant Sci. 157 (1996) 703-712.

[8] J. Falk, G. Andersen, B. Kernebeck, K. Krupinska, Constitutive overexpression of barley 4-hydroxyphenylpyruvate dioxygenase in tobacco results in elevation of the vitamin $\mathrm{E}$ content in seeds but not in leaves, FEBS Lett. 540 (2003) 35-40.

[9] J. Falk, A. Krahnstover, T.A. van der Kooij, M. Schlensog, K. Krupinska, Tocopherol and tocotrienol accumulation during development of caryopses from barley (Hordeum vulgare L.), Phytochemistry 65 (22) (2004) 2977-2985.

[10] J. Garcia-Plazaola, J.M. Becerril, Seasonal changes in photosynthetic pigments and antioxidants in beech (Fagus sylvatica) in a Mediterranean climate: implications for tree decline diagnosis, Aust. J. Plant Physiol. 28 (2001) 225-232.

[11] G.S. Hall, D.L. Laidman, The pattern and control of isoprenoid quinone and tocopherol metabolism in the germinating grain of wheat (Triticum vulgare), Biochem. J. 108 (1967) 475-482.

[12] G. Halliwel, J. Gutteridge, Free Radicals in Biology and Medicine, Clarendon Press, Oxford, 1989.

[13] D. Hofius, U. Sonnewald, Vitamin E biosynthesis: biochemistry meets cell biology, Trends in Plant Sci. 8 (2003) 6-8.

[14] G. Horvath, L. Wessjohann, Y. Guisez, E. Biebaut, R.J. Caubergs, N. Horemans, Seeds of grapes of Vitis vinifera var. alphonse lavallee 
(Royal) a possible model tissue for studying tocotrienol biosynthesis, Acta Hortic. 652 (2004) 415-424.

[15] A. Kamal-Eldin, L. Appelqvist, The chemistry and antioxidant properties of tocopherols and tocotrienols, Lipids 31 (1996) 671-701.

[16] B. Karunanandaa, Q. Qi, M. Hao, S.R. Baszis, P.K. Jensen, Y. Wong, J. Jiang, M. Venkatramesh, K.J. Gryus, F. Moshiri, D. PostBeittenmiller, J.D. Weiss, H.E. Valentin, Metabolically engineered oilseed crops with enhanced seed tocopherol, Metab. Eng. 7 (2005) 384400.

[17] C. Lubrano, J.R. Robin, A. Khaiat, Fatty acid and sterol and tocopherol composition of oil from the fruit mesocarp of six palm species in French Guinea, Oleagineux 49 (1994) 59-65.

[18] S. Munné-Bosch, L. Alegre, The function of tocopherols and tocotrienols in plants, Crit. Rev. Plant Sci. 21 (2002) 31-57.

[19] C. Peisker, T. Duggelin, D. Rentsch, P. Matile, Phytol and the breakdown of chlorophyll in scenescent leaves, J. Plant Physiol. 135 (1989) 428-432.

[20] G.W. Piironen, K.R. Price, M.J.C. Rhodes, G. Wiliamsons, Tocopherols and tocotrienols in Finnish foods: vegetables, fruits and berries, J. Agric. Food Chem. 34 (1986) 742-746.

[21] C. Pratt, Reproductive anatomy in cultivated grapes-a review, Am. J. Enol. Vitic. 22 (1971) 92-109.

[22] P. Rippert, C. Scimemi, M. Dubald, M. Matringe, Engineering plant shikimate pathway for production of tocotrienol and improving herbicide resistance, Plant Physiol. 134 (1) (2004) 92-100.

[23] M.A. Simontacchi, C.G. Caro, S. Fraga, Puntarulo, Oxidative stress affects $\alpha$-tocopherol content in soybean embryonic axes upon imbibition and following germination, Plant Physiol. 103 (3) (1993) 949-953.

[24] S.E. Sattler, E.B. Cahoon, S.J. Coughlan, D. DellaPenna, Characterization of tocopherol cyclases from higher plants and cyanobacteria. Evolutionary implications for tocopherol synthesis and function, Plant Physiol. 132 (2003) 2184-2195.

[25] S.E. Sattler, L.U. Gilliland, M. Magallanes-Lundback, M. Pollard, D. DellaPenna, Vitamin E Is essential for seed longevity and for preventing lipid peroxidation during germination, Plant Cell 16 (2004) 14191432.

[26] B. Savidge, J.D. Weiss, Y.H. Wong, M.W. Lassner, T.A. Mitsky, C.K. Shewmaker, D. Post-Beittenmiller, H.E. Valentin, Isolation and charac- terization of homogentisate phytyltransferase genes from Synechocystis sp. PCC 6803 and Arabidopsis, Plant Physiol. 129 (2002) 321-332.

[27] M. Schledz, A. Siedler, P. Beyer, G. Neuhaus, A novel phytyltransferase from Synechocystis sp. PCC 6803 involved in tocopherol biosynthesis, FEBS Lett. 15 (2001) 15-20.

[28] G. Schultz, Biosynthesis of $\alpha$-tocopherol in chloroplasts of higher plants, Fat Sci.Technol. 92 (1990) 86-91.

[29] J. Soll, M. Kemmerling, G. Schultz, Tocopherol and plastoquinone synthesis in spinach chloroplasts subfractions, Arch. Biochem. Biophys. 204 (1980) 544-550.

[30] J. Soll, G. Schultz, J. Joyard, R. Douce, M.A. Block, Localisation and synthesis of prenylquinones in isolated outer and inner envelope membranes from spinach chloroplasts, Arch. Biochem. Biophys. 238 (1985) 290-299.

[31] D.R. Threlfall, The biosynthesis of vitamin E and $\mathrm{K}$ and related compounds, Vitam. Horm. 29 (1971) 153-200.

[32] W.A. Tramontano, D. Ganci, M. Pennino, E.S. Dierenfeld, Age dependent $\alpha$-tocopherol concentrations in leaves of soybean and pinto beans, Phytochemistry 31 (1992) 3349-3351.

[33] H.I. Uzun, Seasonal variability in berry parameters of grape cultivars under subtropical conditions, Acta Hortic. 441 (1997) 387-394.

[34] H.E. Valentin, Q. Qi, Biotechnological production and application of vitamin E: current state and prospects, Appl. Microbiol. Biotechnol. 68 (2005) 436-444.

[35] A.L. Van Eenennaam, K. Lincoln, T.P. Durrett, H.E. Valentin, C.K. Shewmaker, G.M. Thorne, J. Jiang, S.R. Baszis, C.K. Levering, E.D. Aasen, M. Hao, J.C. Stein, S.R. Norris, R.L. Last, Engineering vitamin E content: from Arabidopsis mutant to soy oil, Plant Cell 15 (2003) 3007-3019.

[36] P.R. Walker, Z. Chen, L.I. Técsi, F. Famiani, P.J. Lea, R.C. Leegood, Phosphoenolpyruvate carboxykinase plays a role in interactions of carbon and nitrogen metabolism during grape seed development, Planta 210 (1999) 9-18.

[37] C. Wu, H. Washida, Y. Onodera, K. Harada, F. Takaiwa, Quantitative nature of the prolamin-box, ACGT and AACA motifs in a rice glutelin gene promoter: minimal cis-element requirements for endosperm specific gene expression, Plant J. 23 (2000) 415-421. 